\title{
La responsabilidad social empresarial y el desarrollo sostenible en latinoamerica en tiempos de pandemia
}

\author{
William Segundo Valencia \\ will_sv@hotmail.com \\ Universidad Cesar Vallejo \\ https://orcid.org/0000-0002-1349-6370 \\ Mercedes Janqui Esquivel \\ mercedesjanqui@gmail.com \\ Universidad Cesar Vallejo \\ https://orcid.org/0000-0001-9844-9225
}

\section{RESUME}

La pandemia del coronavirus (COVID-19) trajo consigo severas consecuencias de tipo económico, social y ambiental. En el mundo, diversas empresas se vieron en la necesidad de renovar sus modelos de intervención, con la finalidad de poder garantizar su continuidad en el mercado. Producto de ello, se abre la posibilidad de implementar la Responsabilidad Social Empresarial (RSE) como un instrumento innovador de gestión institucional. Por lo tanto, el objetivo del presente estudio fue analizar el impacto de la RSE como estrategia empresarial para hacer frente a las consecuencias de la pandemia en Latinoamérica. Se utilizó la metodología PRISMA, desarrollando la revisión sistemática de veintiún artículos de investigación de las principales bases de datos científicas: Scielo, Redalyc, Dialnet, Ebsco y Google Académico. Se concluye que la implementación de la RSE está directamente relacionada a una mayor productividad, rentabilidad y sostenibilidad como mayores beneficios, además de garantizar una estrecha relación positiva con el medio ambiente para su preservación y conservación.

Palabras clave: coronavirus; gestión; sostenibilidad; productividad; rentabilidad. 


\title{
Corporate social responsibility and sustainable development in Latin America in times of pandemic
}

\begin{abstract}
The coronavirus pandemic (COVID-19) brought severe economic, social and environmental consequences. In the world, various companies found it necessary to renew their intervention models, in order to guarantee their continuity in the market. As a result, the possibility of implementing Corporate Social Responsibility (CSR) as an innovative instrument of institutional management opens. Therefore, the objective of this study was to analyze the impact of CSR as a business strategy to face the consequences of the pandemic in Latin America. The PRISMA methodology was used, developing the systematic review of twenty-one research articles from the main scientific databases: Scielo, Redalyc, Dialnet, Ebsco and Google Academic. It is concluded that the implementation of CSR is directly related to greater productivity, profitability and sustainability as greater benefits, in addition to guaranteeing a close positive relationship with the environment for its preservation and conservation.
\end{abstract}

Keywords: Coronavirus, management, sustainability, productivity, profitability.

Artículo recibido: 30 noviembre. 2021 Aceptado para publicación: 29 diciembre 2021

Correspondencia: will_sv@,hotmail.com

Conflictos de Interés: Ninguna que declarar 


\section{INTRODUCCIÓN}

La pandemia del coronavirus (COVID-19) aterrizó en Latinoamérica en el momento más crítico desde el punto de vista social, económico, y medioambiental (CEPAL, 2020). Esta crisis sanitaria produjo contracciones en los grandes mercados económicos de las potencias mundiales como en los Estados Unidos, la Unión Europea y China (Quevedo, 2020) así como la interrupción de las principales cadenas de valor (García et al., 2020). Latinoamérica no es la excepción; también hace frente a una crítica situación económica, acompañada además de un gran descontento social (Eclac, 2020; OECD, 2020). La reducción financiera sobre los mercados de trabajo mundial; se ve reflejada en un incremento masivo del desempleo a nivel mundial (Gamero et al., 2020; OIT, 2020). Durante el año 2020, se estimó la reducción de un $8.8 \%$ respecto a la cantidad de horas de trabajo (en comparación al cuarto trimestre de 2019), lo que significa la pérdida de 255 millones de empleos a tiempo completo (OIT, 2021).

Actualmente, el impacto ocasionado en las organizaciones empresariales de la región, muestran una acelerada disminución de productividad y desarrollo de actividades en los principales sectores económicos como el turismo, manufactura; comercio, transporte (Banco Mundial, 2020); otro efecto colateral es el incremento de tasas de informalidad como en Brasil, México, Bolivia, Colombia, Ecuador, Chile y Venezuela (Eclac, 2020; López, 2020). Perú, Chile, Bolivia, Colombia y Ecuador son los países más afectados en las exportaciones con destino a Asia (Peñafiel-Chang et al., 2020). Las medidas establecidas por los gobiernos para la contención de la propagación de la enfermedad, también tienen consecuencias; las cuarentenas aplicadas, el cierre de fronteras; y las intermisiones del comercio impactan directamente en la producción y distribución de alimentos, disminuyendo el acceso libre y fácil de la población a las principales fuentes de alimentos (Oyague, 2020).

Con respecto al medio ambiente, la pandemia también ha provocado diversos cambios significativos que impactan negativamente en conservación y protección del entorno (Osuna, 2021; Klemeš et al., 2020). Destaca el masivo crecimiento de producción y consumo de plásticos (Flores, 2020), ocasionado por la alta demanda de equipos de protección personal (EPP), como mascarillas, protectores faciales, batas, guantes, desinfectante de manos en botella, entre otros (Saadat et al., 2020). Por otro lado, como resultado de las medidas de bloqueo, junto a los estrictos protocolos de higiene, la 
mayoría de restaurantes pasaron a ofrecer servicios de comida para llevar y entrega, haciendo uso de grandes cantidades de envases de plástico de un solo uso (Wittmann y Dantas, 2020). Los servicios de venta en línea también han experimentado un aumento en la demanda del uso de productos empacados en plástico de un solo uso. Si bien, todas estas medidas adoptadas juegan un papel importante en la prevención de la propagación de la enfermedad, a corto plazo, el incremento de uso de estos artículos puede contrarrestar los esfuerzos de la humanidad para frenar la contaminación plástica (AEMA, 2020) y control de residuos (Olmedo y Ceberio de León, 2020); el impacto y las consecuencias se verán reflejadas en el medio ambiente y el clima (Sánchez-Gutierrez, 2020), a través de mayores emisiones de gases de efecto invernadero, por consiguiente, el incremento de la contaminación atmosférica (Pérez-Rodrigo et al., 2020).

Todos los países de Latinoamérica hacen frente a los diversos desafíos de la pandemia; con la finalidad de proteger a su población y buscar el reinicio de sus economías, orientando el desarrollo hacia el futuro, cuyo objetivo no solo es volver al crecimiento económico-social, sino también alcanzar los Objetivos de Desarrollo sostenible para la humanidad (Castillo et al., 2021). Lo que abre puerta a la necesidad de aplicar estrategias relacionadas a la Responsabilidad Social Empresarial (RSE), considerada hoy en día, como instrumento que garantiza la sustentabilidad y sostenibilidad de los recursos naturales (Vallejo-Montoya, 2020), asociada a un incremento de rentabilidad con beneficios que se traducen en mejoras para la sociedad frente a aspectos ambientales, culturales y sociales (Vásquez-Giler et al., 2020), así como fortalecer los lazos con los grupos de interés o stakeholders (Nan, 2020); lo que contribuirá a estabilizar los mercados económicos (Pérez et al., 2016).

Debemos ver la complementariedad que existe entre la entre la RSE y la Sostenibilidad (Olaya, 2020). Su implementación, favorece el desarrollo de operaciones de forma sustentable en lo económico, lo social y lo ambiental (Remache-Rubio, 2018), además de encaminar el cumplimiento de: buen gobierno corporativo y transparente, políticas de personal que respeten sus derechos y favorezcan su desarrollo, ética con el consumidor, protección del medio ambiente y acciones de protección social (Camarán at al., 2019). Por ende, las empresas contribuyen a la sostenibilidad siendo socialmente responsable. Desde el aspecto económico, aporta a la generación de empleos y riqueza, satisfaciendo necesidades de sociedad. Desde el aspecto ambiental, protege los recursos y reduce su 
huella de carbono y residuos. Desde el aspecto social, vela por los derechos humanos, por sus colaboradores, así como de las comunidades más vulnerables mediante programas específicos de protección (Shangzhi, 2021). Podemos concluir que la Responsabilidad social es uno de los medios y la Sostenibilidad es el fin al que quiere arribar la sociedad. Por ello, en función a lo expuesto, el objetivo de la presente investigación fue: analizar el impacto de la RSE como estrategia para afrontar las consecuencias de la pandemia del COVID-19 en Latinoamérica. Sobre este tema, se plantearon dos objetivos específicos: el primero fue analizar las estrategias y enfoques metodológicos de las investigaciones seleccionadas. El segundo fue analizar los conceptos y beneficios de la implementación de la RSE en las organizaciones; alineados al desarrollo sostenible en Latinoamérica.

\section{METODOS}

El presente estudio realizó una revisión sistemática de literatura (RSL), este es un procedimiento utilizado para generar un análisis crítico reflexivo del contenido de los documentos científicos que serán utilizados en la elaboración de esta investigación (García-Peñalvo, 2020). En ese entender, como primer paso se realizó la búsqueda minuciosa de artículos originales de la variable Responsabilidad Social Empresarial (RSE) a partir de artículos publicados en los años 2020 y 2021 en los siguientes repositorios de datos: DIALNET, EBSCO, SCIELO, REDALYC y GOOGLE ACADEMICO. Las palabras clave que guiaron la revisión fueron "Responsabilidad Social Empresarial" y "Corporate social responsibility" ambas delimitadas en Latinoamérica, donde se centra el estudio. De esta revisión, se obtuvieron ciento siete (107) artículos y revisiones, sin distinción idiomas, de ellos se descartaron aquellos que no contaban con los criterios de inclusión: conceptos de RSE alineados al Desarrollo Sostenible, estudios desarrollados en Latinoamérica, investigaciones publicadas en español e inglés y artículos de acceso abierto. Como resultado, de acuerdo con el método Prisma (Hutton et al., 2016) se obtuvo un total de veintiún (21) artículos, los cuales cumplen con los objetivos propuestos y constituyen la base para la presente revisión. 


\section{Figura 1}

Proceso de revisión PRISMA

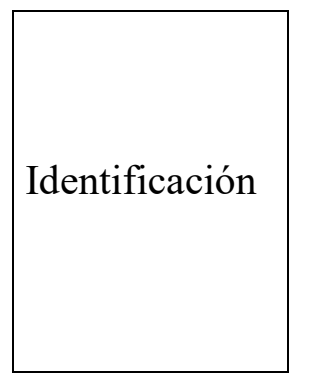

De las siguientes bases de datos: SCIELO, REDALYC, DIALNET, EBSCO y GOOGLE ACADEMICO se registraron 107 artículos, todos ellos referidos a "Responsabilidad Social Empresarial" y "Corporate social responsibility" dentro del periodo 2020-2021

\section{Revisión}

De los 107 artículos, solo quedaron 96 por contener los conceptos de RSE alineados al Desarrollo Sostenible.

Elegibilidad

De los 96 artículos, solo quedaron 21 que se encontraban dentro de la región Latinoamérica.

Inclusión

De los 21 artículos, cumplieron con los objetivos 21 artículos

\section{Tabla 1}

Resultados de selección en los repositorios

\begin{tabular}{|c|c|c|c|c|c|c|c|c|}
\hline Repositorio & Código & $\begin{array}{c}\text { Combinación de } \\
\text { búsqueda }\end{array}$ & Resultados & $\begin{array}{c}\text { Temática } \\
\text { (conceptos } \\
\text { de RSP) }\end{array}$ & Región & Acceso & Objetivos & $\begin{array}{l}\text { Artículos } \\
\text { no } \\
\text { repetidos }\end{array}$ \\
\hline SCIELO & A1 & $\begin{array}{l}\text { Responsabilidad } \\
\text { Social } \\
\text { Empresarial o } \\
\text { Corporate social } \\
\text { responsibility }\end{array}$ & 31 & 7 & 1 & 1 & 1 & 1 \\
\hline REDALYC & B1 & $\begin{array}{l}\text { Responsabilidad } \\
\text { Social } \\
\text { Empresarial o } \\
\text { Corporate social }\end{array}$ & 36 & 8 & 5 & 2 & 5 & 2 \\
\hline
\end{tabular}




\begin{tabular}{|c|c|c|c|c|c|c|c|c|}
\hline & & responsibility & & & & & & \\
\hline DIALNET & $\mathrm{C} 1$ & $\begin{array}{l}\text { Responsabilidad } \\
\text { Social } \\
\text { Empresarial o } \\
\text { Corporate social } \\
\text { responsibility }\end{array}$ & 18 & 12 & 6 & 5 & 5 & 5 \\
\hline EBSCO & D1 & $\begin{array}{l}\text { Responsabilidad } \\
\text { Social } \\
\text { Empresarial o } \\
\text { Corporate social } \\
\text { responsibility }\end{array}$ & 11 & 4 & 0 & 0 & 0 & 0 \\
\hline $\begin{array}{l}\text { GOOGLE } \\
\text { ACADEMI } \\
\mathrm{CO}\end{array}$ & E1 & $\begin{array}{l}\text { Responsabilidad } \\
\text { Social } \\
\text { Empresarial o } \\
\text { Corporate social } \\
\text { responsibility }\end{array}$ & 11 & 65 & 20 & 13 & 13 & 13 \\
\hline Total & & & 107 & 96 & 32 & 21 & 24 & 21 \\
\hline
\end{tabular}

En la tabla 1, se aprecia el proceso de selección; donde en función a los repositorios elegidos y los criterios de búsqueda aplicados (que fue "Responsabilidad Social Empresarial" y "Corporate social responsibility", se pudieron encontrar ciento siete (107) artículos. Sin embargo, al tomar en cuentas los criterios de inclusión formulados al inicio de la metodología, respecto a la región de aplicación (Latinoamérica), y su vinculación con los objetivos se seleccionaron solo veintiún (21) investigaciones.

\section{RESULTADOS}

En función a los objetivos formulados, se revisaron los veintiún (21) artículos seleccionados.

\subsection{Características de los artículos}

De acuerdo con el proceso de revisión prisma, podemos observar que de las veintiún (21) fuentes seleccionadas en la presente revisión, la mayoría de trabajos publicados en Latinoamérica, se encuentra en Ecuador, con diez (10) investigaciones, seguida de México con cinco (5), luego Colombia con tres (3), y finalmente; Chile, Perú y Cuba, países en los que se ha desarrollado una (1) investigación en cada región.

Tabla 2 Artículos incluidos en la revisión

$\begin{array}{lll}\text { Repositorio } & \text { Autor, año } & \text { País }\end{array}$




\begin{tabular}{lll}
\hline SCIELO & (Vilca et al., 2021) & Perú \\
REDALYC & (Ormaza et al., 2020) & Ecuador \\
REDALYC & (Fong et al., 2020) & México \\
DIALNET & (Ávila y Cedeño, 2020) & Ecuador \\
DIALNET & (Pérez, 2020) & México \\
DIALNET & (Salas et al., 2020) & Colombia \\
DIALNET & (Véliz y Zaldumbide, 2021) & Ecuador \\
DIALNET & (Palomeque et al., 2021) & Ecuador \\
GOOGLE ACADEMICO & (Ríos et al., 2020) & Ecuador \\
GOOGLE ACADEMICO & (Espinoza et al., 2020) & Ecuador \\
GOOGLE ACADEMICO & (Yauri et al., 2020) & Ecuador \\
GOOGLE ACADEMICO & (Huerta y Andrade, 2021) & México \\
GOOGLE ACADEMICO & (Severino et al., 2020) & Chile \\
GOOGLE ACADEMICO & (Lara y Sánchez, 2021) & México \\
GOOGLE ACADEMICO & (Pineda et al., 2020) & Ecuador \\
GOOGLE ACADEMICO & (Dotres y Sánchez, 2020) & Cuba \\
GOOGLE ACADEMICO & (Petro et al., 2020) & Colombia \\
GOOGLE ACADEMICO & (Pérez et al., 2020) & Colombia \\
GOOGLE ACADEMICO & (Navarrete et al., 2021) \\
GOOGLE ACADEMICO & (Vergara et al., 2021) \\
GOOGLE ACADEMICO & $\quad$ (Pilay et al., 2021) & Ecuador \\
\hline & & \\
GOador
\end{tabular}

Figura 2 Porcentaje de fuentes de acuerdo con la región de procedencia

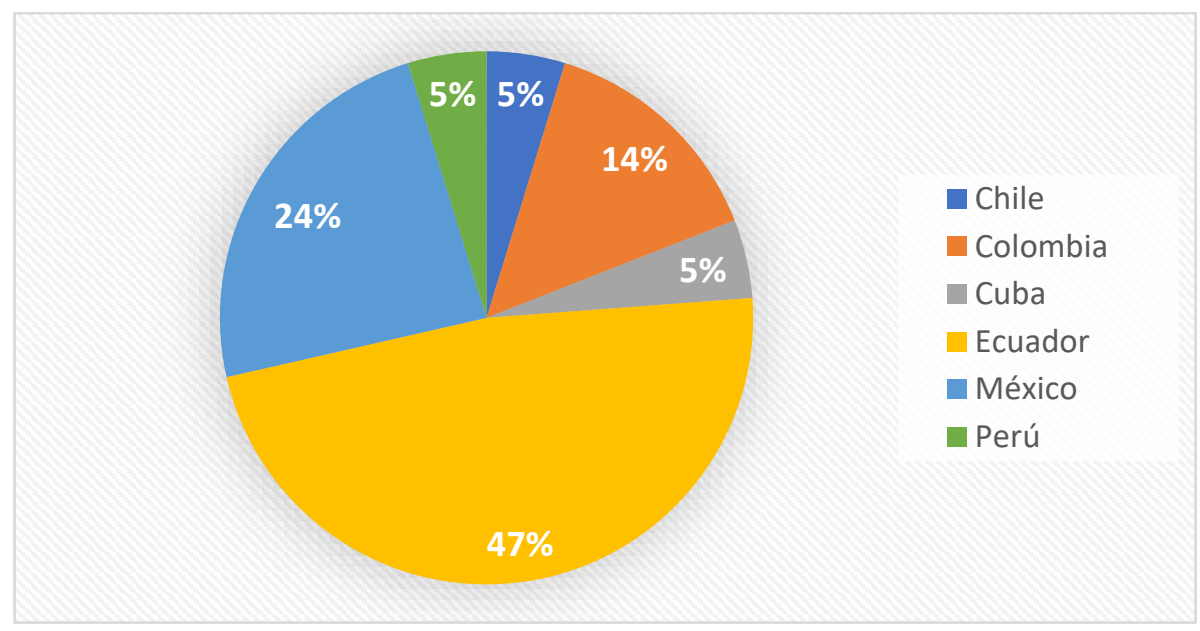


En la figura 2, se puede observar la procedencia del origen de los artículos de acuerdo al país en el cual se publicó. De los cuales, el 47 \% de artículos procede de Ecuador, seguido de Colombia con 24 \% y México con 14\%. Siendo los países con mayores publicaciones de acceso libre que se presenta en la investigación.

Tabla 3 Estrategias y enfoques metodológicos

\begin{tabular}{llc}
\hline \multicolumn{1}{c}{ Autor, año } & Enfoques & Instrumento de recolección de datos \\
\hline (Vilca et al., 2021) & Cualitativo & Revisión documental \\
(Ormaza et al., 2020) & Cualitativo & Revisión documental \\
(Fong et al., 2020) & Mixto & Análisis de contenido \\
(Ávila y Cedeño, 2020) & Cuantitativo & Entrevistas \\
(Pérez, 2020) & Cualitativo & Revisión documental \\
(Salas et al., 2020) & Cuantitativo & Entrevistas \\
(Véliz y Zaldumbide, 2021) & Mixto & Encuestas \\
(Palomeque et al., 2021) & Cuantitativo & Entrevistas \\
(Ríos et al., 2020) & Mixto & Entrevistas \\
(Espinoza et al., 2020) & Mixto & Revisión documental \\
(Yauri et al., 2020) & Mixto & Encuestas \\
(Huerta y Andrade, 2021) & Cuantitativo & Análisis de contenido \\
(Severino et al., 2020) & Cuantitativo & Encuestas \\
(Lara y Sánchez, 2021) & Cuantitativo & Encuestas \\
(Pineda et al., 2020) & Cuantitativo & Encuestas \\
(Dotres y Sánchez, 2020) & Mixto & Revisión documental, encuestas y entrevistas \\
(Petro et al., 2020) & Cuantitativo & Entrevistas \\
(Pérez et al., 2020) & Cualitativo & Revisión documental y entrevistas \\
(Navarrete et al., 2021) & Cuantitativo & Encuestas \\
(Vergara et al., 2021) & Cuantitativo & Revisión documental \\
(Pilay et al., 2021) & Cuantitativo & Encuestas \\
\hline
\end{tabular}

\section{Figura 3}

Porcentaje de fuentes de acuerdo con la metodología utilizada 


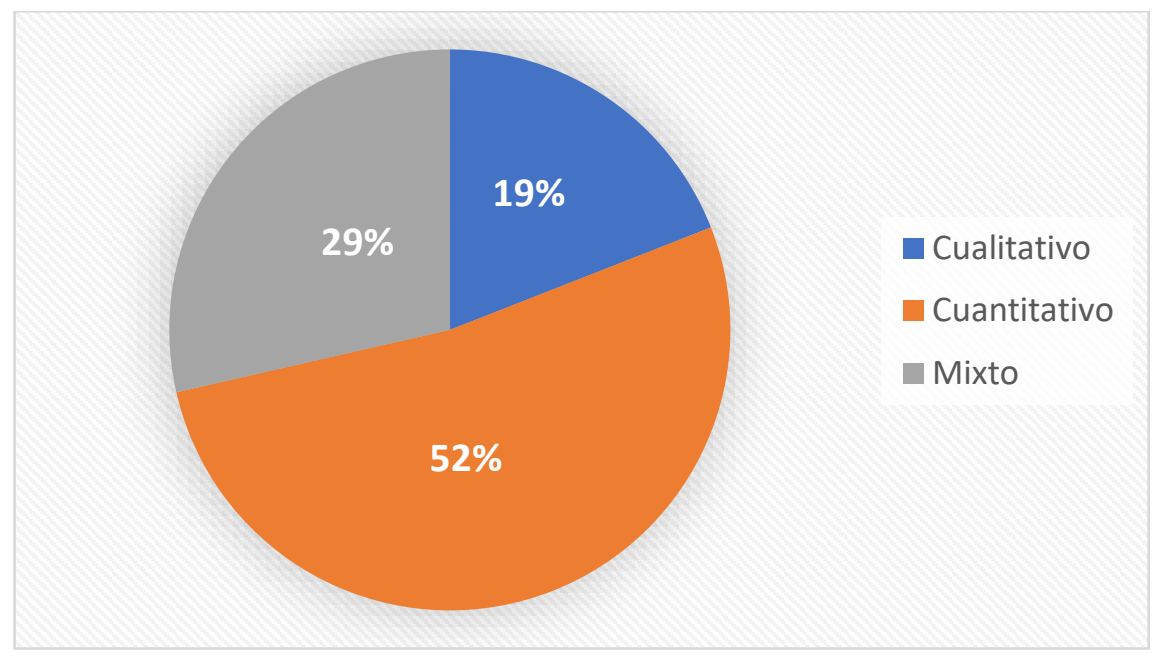

En la figura 3, se puede observar que el 52\% de los artículos analizados, aplicaron el enfoque cualitativo, $29 \%$ utilizaron un enfoque mixto en sus investigaciones, y solamente el 19\% fueron de un enfoque cualitativo.

\section{Figura 4}

Instrumentos de recolección de datos

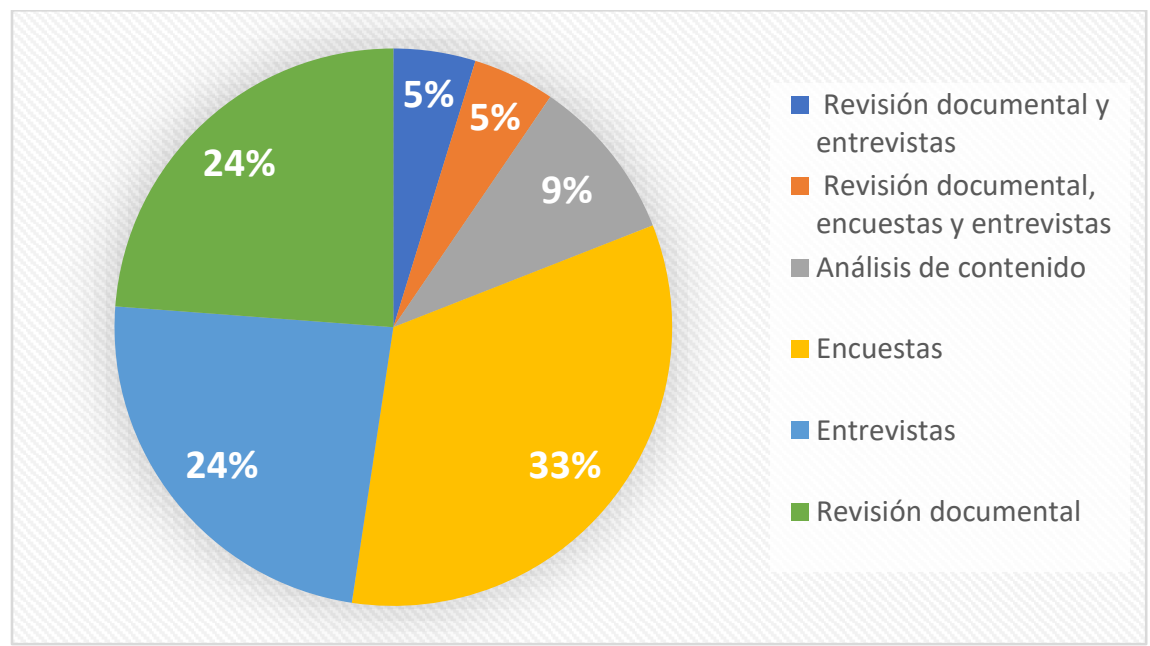

En la figura 4, podemos observar que, dentro de los instrumentos para la recolección de datos utilizados por los artículos seleccionados, predomina las encuestas, entrevistas y la revisión documental.

\subsection{Conceptos y beneficios de la implementación de la RSE en las organizaciones}

En las fuentes de información revisadas, se encontró una gran diversidad de conceptos y definiciones, y como consecuencia de la pandemia, se vienen innovando diversos enfoques de aplicación, con el único propósito de buscar que las empresas sean responsables y sostenibles con el medio ambiente, además de obtener rentabilidad 
económica como beneficio. Por ello, definen a la Responsabilidad Social Empresarial (RSE) como un instrumento que favorecerá que las empresas logren maximizar sus beneficios económicos y su capacidad productiva (Véliz y Zaldumbide, 2021); así como fortalecer la relación positiva entre la sociedad y el medio ambiente, mediante la inversión de tecnología limpia en el capital humano (Fong et al., 2020). De igual manera, la sostenibilidad, es considerado un factor clave para la ejecución de mejoras en las empresas por medio del enfoque del Triple Balance, donde se incluye: a) la dimensión social, orientada a mejorar la calidad de vida, salud y educación; b) dimensión económica, busca el crecimiento equitativo sin perjuicio de los recursos naturales; y, c) dimensión ambiental, uso constante de energía renovable y la protección permanente de lanaturaleza, (Ormaza et al., 2020). La RSE es considerado un paradigma de organización y trabajo que permite retribuir a la comunidad lo que la empresa "toma de ella"; entendida como la forma perfecta de hacer negocio de manera sustentable (Pineda et al., 2020).

Si bien las empresas son impulsoras de actividad y actualmente son las que generan gran parte del empleo para la población, también tienen responsabilidad social; la cual va ligada a la innovacion; para mantener una posición competitiva dentro de la economía mundial globalizada (Navarrete et al., 2020), con esto; se logra no únicamente contribuir aún más con la sociedad, sino también incrementar la productividad y el crecimiento organizacional (Pérez, 2020). Innovar garantiza la permanencia de estas en el mercado, obteniendo beneficios como la fidelización de los clientes, mayor productividad del personal y la satisfacción de la comunidad en general (Pilay et al., 2021). La innovación requiere la participación de personas con diferentes capacidades; es un proceso es interdisciplinario; el éxito radica en las relaciones entre los trabajadores; en el conocimiento tácito y en la capacidad de la institución en transformarlo en conocimiento explícito; es decir, en el recurso humano (Fong et al., 2020).

Por otra parte, la RSE es una estrategia rentable que debe ser incorporada en las empresas, que buscan posicionar su marca en el mercado, innovar la imagen corporativa, captar la preferencia de los grupos de interés (stakeholders) y fomentar el equilibrio entre la empresa y la sociedad en la que opera (Dotres y Sánchez, 2020). Sin embargo, también son muy pocas las empresas que incorporan la RSE, debido a que su implementación implica la generación de costos adicionales que disminuyen los beneficios de la firma, y, por tanto, su eficiencia económica (Lara y Sánchez, 2021). Ganar una buena reputación 
corporativa, según lógica pura, supondrá a futuro, mayor rentabilidad para la empresa; por ende, la implementación de la RSE, será exitoso siempre y cuando exista talento humano capaz y competente que pueda aportar al desarrollo de actividades con calidad, efectividad y confianza (Petro et al., 2020).

La necesidad de implementar la RSE, surge a consecuencia de las deficientes condiciones en las que encontraban diversas empresas y organizaciones, ya que, al estar enfrascados solamente en la búsqueda constante de producir bienes y servicios, para producir ganancias, establecen el fenómeno de producción en masa (Vergara et al., 2020). Por lo tanto, una empresa se considera socialmente responsable, cuando además de ofrecer productos y servicios de calidad a la sociedad, es capaz de generar empleos y utilidades, además de pagar impuestos (Yauri et al., 2020); asimismo, dentro del territorio de intervención, muestra interés en identificar los diversos problemas que puedan afectar al desarrollo de la población y como consecuencia, también propone alternativas para su solución (Huerta y Andrade, 2021).

Otro aspecto de vital importancia que se ha identificado, es el concepto de los grupos de interés o más denominado "Skateholders" (Zárate et al., 2020). Estos grupos contribuyen directamente al logro de los objetivos de una organización o también pueden verse afectados por ella (Vilca et al., 2021). En ellos, destacan: los directivos, los trabajadores y sus familias, los inversionistas, los clientes, los proveedores, el estado y sus organismos públicos, ONG, academias, medios de comunicación, instituciones científicas, entre otras (Ríos et al., 2020). La RSE fomenta el compromiso y seguridad de los grupos de interés (Perez et al.,2020), mediante el apoyo social, la protección ambiental y la mejora de las condiciones laborales (Ávila y Cedeño, 2020), por ende, las organizaciones empresariales deben favorecer el desarrollo comunitario, mediante la generación de puestos de trabajo en sus áreas de operación (Salas, 2020); este modelo de gestión asume mecanismos de producción más limpia para la sociedad (Espinoza et al., 2020), así como las acciones de reciclaje, uso eficiente de la energía; y control y disposición de residuos, generara ingresos extras y en consecuencia, satisfacción del usuario (Palomeque et al., 2021). Por último, ofrece alternativas a visionar y contribuir a la mejora continua de los elementos de la calidad en la prestación de servicios a la sociedad y al desempeño laboral (Severino et al., 2020). 
Durante el análisis de los trabajos de investigación, se pudo resumir el impacto de la RSE en las organizaciones, incluye diversos beneficios para las mismas. Entre los cuales se pueden destacar la sostenibilidad, la innovación, el desempeño organizacional, el compromiso organizacional y la satisfacción de los grupos de interés entre otros.

Tabla 4 Impacto de la RSE en las organizaciones

\begin{tabular}{|c|c|c|}
\hline Autor, año & País & Beneficios \\
\hline (Vilca et al., 2021) & Perú & $\begin{array}{l}\text { Manejo de conflictos y satisfacción de los } \\
\text { grupos de interés }\end{array}$ \\
\hline (Ormaza et al., 2020) & Ecuador & Sostenibilidad y equilibrio financiero \\
\hline (Fong et al., 2020) & Chile & Innovación y rentabilidad \\
\hline (Ríos et al., 2020) & Ecuador & $\begin{array}{l}\text { Sostenibilidad, } \\
\text { organizacional y satisfacción de los } \\
\text { grupos de interés }\end{array}$ \\
\hline (Ávila y Cedeño, 2020) & Ecuador & $\begin{array}{l}\text { Sostenibilidad y satisfacción de los } \\
\text { grupos de interés }\end{array}$ \\
\hline (Pérez, 2020) & México & Innovación y productividad \\
\hline (Salas, 2020) & Colombia & Prevención de conflictos \\
\hline (Espinoza et al., 2020) & Ecuador & $\begin{array}{l}\text { Sostenibilidad, equilibrio financiero y } \\
\text { satisfacción de los grupos de interés }\end{array}$ \\
\hline (Yauri et al., 2020) & Ecuador & $\begin{array}{l}\text { Satisfacción de los grupos de interés y } \\
\text { sostenibilidad }\end{array}$ \\
\hline $\begin{array}{l}\text { (Véliz y Zaldumbide, } \\
\text { 2021) }\end{array}$ & Ecuador & $\begin{array}{l}\text { Sostenibilidad, productividad } \\
\text { desempeño organizacional }\end{array}$ \\
\hline (Huerta y Andrade, 2021) & México & Compromiso organizacional \\
\hline (Severino et al., 2020) & Chile & Satisfacción de los grupos de interés \\
\hline (Lara y Sánchez, 2021) & México & $\begin{array}{l}\text { Competitividad, rentabilidad corporativa, } \\
\text { reputación }\end{array}$ \\
\hline (Pineda et al., 2020) & Ecuador & Sostenibilidad y equilibrio financiero \\
\hline (Dotres y Sánchez, 2020) & Cuba & Rentabilidad \\
\hline (Petro et al., 2020) & Colombia & Rentabilidad y Sostenibilidad \\
\hline (Navarrete et al., 2020) & México & Innovacion y rentabilidad \\
\hline (Vergara et al., 2020) & Ecuador & $\begin{array}{l}\text { Rendimiento económico y calidad de } \\
\text { imagen }\end{array}$ \\
\hline (Palomeque et al., 2021) & Ecuador & Satisfacción de los grupos de interés \\
\hline (Pilay et al., 2021) & Ecuador & Rentabilidad y productividad \\
\hline (Pérez et al., 2020) & Colombia & \\
\hline
\end{tabular}

Asimismo, para afrontar la pandemia e impulsar el desarrollo sostenible, podemos mencionar que la implementación de la RES como estrategia organizacional, propone 
acciones de convergencia y sinergia entre las dimensiones social, ambiental y económica, esta combinación, permitirá garantizar la sostenibilidad de cualquier empresa $u$ organización a largo plazo.

Tabla 5 Acciones de sostenibilidad de la RSE en las organizaciones

\begin{tabular}{|c|c|c|c|}
\hline Dimensión & Acciones & Indicador & Autor \\
\hline \multirow{3}{*}{ Social } & $\begin{array}{l}\text { Satisfacción en } \\
\text { clientes }\end{array}$ & $\begin{array}{l}\text { Servicio de atención al } \\
\text { ciudadano }\end{array}$ & $\begin{array}{l}\text { (Vilca et al., 2021) (Ríos et al., } \\
\text { 2020) (Ávila y Cedeño, 2020) }\end{array}$ \\
\hline & Código de ética & Publicidad verdadera & $\begin{array}{l}\text { (Espinoza et al., 2020) (Yauri } \\
\text { et al., 2020) (Huerta y }\end{array}$ \\
\hline & Proyectos sociales & $\begin{array}{l}\text { Programas de apoyo y } \\
\text { beneficio social }\end{array}$ & $\begin{array}{l}\text { Andrade, 2021) (Severino et } \\
\text { al., 2020) (Palomeque et al., } \\
\text { 2021) (Pérez et al., 2020) }\end{array}$ \\
\hline \multirow{3}{*}{ Ambiental } & $\begin{array}{c}\text { Políticas } \\
\text { ambientales y } \\
\text { legales }\end{array}$ & $\begin{array}{l}\text { Estudios de impacto y } \\
\text { normativas de protección } \\
\text { ambiental }\end{array}$ & \multirow{3}{*}{$\begin{array}{l}\text { (Ormaza et al., 2020) (Ríos et } \\
\text { al., 2020) (Pérez, 2020) (Véliz } \\
\text { y Zaldumbide, 2021) (Pineda } \\
\text { et al., 2020) (Petro et al., 2020) } \\
\text { (Navarrete et al., 2020) (Pilay } \\
\text { et al., 2021) }\end{array}$} \\
\hline & $\begin{array}{l}\text { Regulación del } \\
\text { consumo }\end{array}$ & $\begin{array}{l}\text { Reducción de consumo de } \\
\text { agua, energía, combustibles. } \\
\text { Acciones para optimizar } \\
\text { recursos }\end{array}$ & \\
\hline & $\begin{array}{l}\text { Actividades de } \\
\text { reciclaje }\end{array}$ & 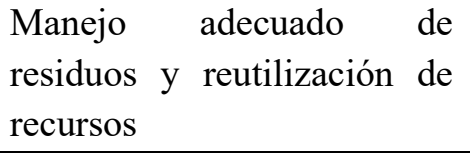 & \\
\hline \multirow{3}{*}{ Económica } & $\begin{array}{l}\text { Obligaciones } \\
\text { legales }\end{array}$ & $\begin{array}{l}\text { Tributos al gobierno y } \\
\text { servicios sociales para } \\
\text { empleados y usuarios }\end{array}$ & $\begin{array}{l}\text { (Ormaza et al., 2020) } \\
\text { (Fong et al., 2020) } \\
\text { (Salas, 2020) (Espinoza et al., }\end{array}$ \\
\hline & $\begin{array}{l}\text { Condiciones de } \\
\text { pagos acordados }\end{array}$ & $\begin{array}{l}\text { Obedecer pagos a grupos de } \\
\text { interés }\end{array}$ & $\begin{array}{l}\text { 2020) (Lara y Sánchez, 2021) } \\
\text { (Pineda et al., 2020) (Dotres y }\end{array}$ \\
\hline & $\begin{array}{l}\text { Control en } \\
\text { operaciones } \\
\text { financieras }\end{array}$ & $\begin{array}{l}\text { Transparencia en la } \\
\text { información, así como } \\
\text { auditorías internas y externas }\end{array}$ & $\begin{array}{l}\text { Sánchez, 2020) (Petro et al., } \\
\text { 2020) (Navarrete et al., 2020) } \\
\text { (Vergara et al., 2020) }\end{array}$ \\
\hline
\end{tabular}

\section{CONCLUSIONES}

La crisis social y económica ocasionada por la enfermedad del coronavirus a nivel mundial, exige a que las empresas $u$ organizaciones adopten nuevas medidas de intervención, así como un cambio estructural y organizativa que permita continuar con sus operaciones y poder garantizar su permanencia del mercado mundial, priorizando el equilibrio entre el bienestar humano y una economía activa, permitiendo en esta nueva realidad de pandemia, una convivencia responsable con el medio ambiente que nos rodea. Como una de las iniciativas para hacer frente a los efectos de la pandemia, se propone a 
la RES como un instrumento innovador de gestión moderna, desde donde se encamina un conjunto de acciones destinadas a atenuar los impactos económicos, ambientales y sociales que causa una empresa en el entorno en que opera. Además, de generar otros beneficios como mayor productividad, rentabilidad, competitividad, fidelización de los clientes, empleados más productivos y empoderados, asegurando de esta forma la sostenibilidad para la empresa y el equilibrio con el medio ambiente y la comunidad; a través de la intervención con tecnología limpia y uso racional de recursos.

\section{REFERENCIAS BIBLIOGRAFICAS}

Ávila, A.C., y Cedeño, M.R. (2020). Las pequeñas y medianas empresas y la responsabilidad social empresarial en el Ecuador. Revista científica dominio de las ciencias, 6(3). https://dialnet.unirioja.es/servlet/articulo? codigo=7539708

AEMA (Agencia Europea del Medio Ambiente). (2020). El coronavirus ha tenido impactos mixtos en el medioambiente. https://www.eea.europa.eu/

Banco Mundial (2020). Respuesta a la crisis del COVID-19: apoyo a la educación terciaria para la continuidad, la adaptación y la innovación. Banco Mundial, Washington, DC.C Banco Mundial. https://openknowledge.worldbank.org/handle/10986/34571Licencia: CC BY 3.0 IGO

Camarán, M.L., Barón M., L., Rueda, S.M.P. (2019). La Responsabilidad social empresarial y los objetivos del desarrollo sostenible (ODS). Revista científica: teoría, enfoques y aplicaciones en las ciencias sociales. ISSN 2610-7910. https://dialnet.unirioja.es/servlet/articulo? codigo $=7013907$

CEPAL (Comisión Económica para América Latina y el Caribe). (2020). El impacto del COVID-19 en América Latina y el Caribe. https://peru.un.org/sites/default/files/2020-

07/SG\%20Policy\%20brief\%20COVID\%20LAC\%20\%28Spanish\%29_10\%20Ju ly_0.pdf\#: :text=crisis\%20cambiarias\%20y\%20de\%20deuda,y\%20p\%C3\%A9r dida $\% 20$ de $\% 20$ biodiver\%E2\%80\%91\%20sidad.

Castillo, C. A., Martínez Puma, E., y Gonzales Portugal, N. (2021, 20 abril). Pandemia por COVID-19 y Objetivos de Desarrollo Sostenible al 2020 COVID-19. Ciencia Latina Revista Cientifica Multidisciplinar. https://doi.org/10.37811/cl_rcm.v5i2.373. 
Dotres, S. y Sánchez, N. (2020). Integración de la responsabilidad social empresarial en la gestión de riesgo en inversiones constructivas. Avances, 22(2), 170-182. http://www.ciget.pinar.cu/ojs/index.php/publicaciones/article/view/529

Eclac (Economic Commission for Latin America and the Caribbean). (2020). Report on the economic impact of coronavirus disease (COVID19) on Latin America and the Caribbean: Study prepared by the ECLAC. Santiago. http://hdl.handle.net/11362/45603

Espinoza, L., Fariño, J. S., Espinoza, Y. P., y Mayorga, M. (2020). Responsabilidad Social y Ambiental: Tratamiento y Disposición Final de Llantas Usadas en la Ciudad de Machala. Gestión En El Tercer Milenio, 23(45), 39-48. https://doi.org/10.15381/gtm.v23i45.17405

Flores, P. (2020). La problemática del consumo de plásticos durante la pandemia de la COVID-19. Southsustainability, 1(2). https://doi.org/10.21142/10.21142/SS0102-2020-016

Fong, C., Parra, A., Soriano, L., y Teodoro, E. (2020). El estado actual de la Responsabilidad Social Empresarial en México. Revista Academia \& Negocios, $6(1), 41-56$.

ISSN:

0719-6245. https://www.redalyc.org/articulo.oa?id=560863786007

García-Peñalvo, F. J. (2020). Método para la revisión sistemática de literatura. Recursos docentes de la asignatura Procesos y Métodos de Modelado para la Ingeniería Web $y$ Web Semántica. Universidad de Salamanca https://repositorio.grial.eu/handle/grial/1961

García, J.A., Morales, T., y Olivares, H.R. (2020). La responsabilidad social empresarial frente al reto de la covid-19. Horizontes de la Contaduría en las Ciencias Sociales, 7(13), 59-84. https://revistahorizontes.uv.mx/index.php/horizont/article/view/30 Gamero, J., y Pérez, J. (2020). Perú > Impacto de la COVID-19 en el empleo y los ingresos laborales. Nota técnica país. Panorama Laboral en tiempos de la COVID-19. Organización Internacional del Trabajo (OIT). https://www.ilo.org/wcmsp5/groups/public/---americas/---rolima/documents/publication/wcms_756474.pdf 
Hongwei, H., y Lloyd, H. (2020). The impact of Covid-19 pandemic on corporate social responsibility and marketing philosophy. ScienceDirect. https://doi.org/10.1016/j.jbusres.2020.05.030

Huerta, A., y Andrade, M.G. (2021). Responsabilidad social empresarial en la misión, visión y valores de las principales empresas en México. Estudios Sociales. Revista de Alimentación Contemporánea y Desarrollo Regional. 31(57). https://doi.org/10.24836/es.v31i57.1109

Hutton, B., Catalá-López, F., y Moher, D. (2016). La extensión de la declaración PRISMA para revisiones sistemáticas que incorporan metaanálisis en red: PRISMA-NMA Science

Direct. https://linkinghub.elsevier.com/retrieve/pii/S0025775316001512

Klemeš, J., Fan, Y., Tan, R. y Jiang P. (2020). «Minimising the present and future plastic waste, energy and environmental footprints related to COVID-19». Renewable and Sustainable. DOI: 10.1016/j.rser.2020.109883

Lara, I., y Sánchez, J. (2020). Responsabilidad social empresarial para la competitividad de las organizaciones en México. Mercados y Negocios, (43), 97-118. https://doi.org/10.32870/myn.v0i43.7546

López-Feldman A., Chávez C., Vélez M.A, Bejarano H., Chimeli A. B., Féres J., Robalino, J., Salcedo, R., y Viteri, C. COVID-19: impactos en el medio ambiente y en el cumplimiento de los ODS en América Latina. Revista Desarrollo y Sociedad, 86 (1). https://doi.org/10.13043/DYS.86.4

Nan, Y., Tung-Boon, K., Lisong, H., Yongxin, L., y Hang, Y. (2020, 1 noviembre). A bibliometric analysis of corporate social responsibility in sustainable development. ScienceDirect. https://doi.org/10.1016/j.jclepro.2020.122679

Navarrete, A.C., Castillo, A.M., Sandoval, S.K., y Trejo, L. (2021). Responsabilidad social como estímulo de la innovación en las mypes del municipio de San Juan del Río, Querétaro. Revista RELAYN, Micro y pequeña empresa en Latinoamérica, 5(1). https://doi.org/10.46990/relayn.2021.5.1.915

Olaya S. (2020). La Responsabilidad Social Empresarial y los Objetivos de Desarrollo Sostenible como complemento organizacional. Adversia, (24), 1-9. https://revistas.udea.edu.co/index.php/adversia/article/view/343092 
Olmedo, C. y Ceberio de León, I. (2020). BASURA y COVID-19: ¿El nexo que no estamos queriendo ver? Proyección. Estudios Geográficos y de Ordenamiento Territorial. ISSN

1852-0006.

https://revistas.uncu.edu.ar/ojs3/index.php/proyeccion/article/view/3986

Ormaza, J., Ochoa, J., Ramírez, F., y Quevedo, J. (2020). Responsabilidad social empresarial en el Ecuador: Abordaje desde la Agenda 2030. Revista de Ciencias Sociales, 26(3). https://www.redalyc.org/articulo.oa?id=28063519009

Osuna, J.A. (2021). La pandemia coronavirus Covid-19 y salud del medio ambiente. Educere, 25(80),259-262.

ISSN:

1316-4910. https://www.redalyc.org/articulo.oa?id=35666280023

Oyague, E., Yaja, A., y Franco, P. (2020). Efectos ambientales del confinamiento debido a la pandemia de COVID-19: evaluación conceptual y análisis de datos empíricos en Tacna, marzo-abril 2020. Ciencia \&Amp; Desarrollo, (26), 2-19. https://doi.org/10.33326/26176033.2020.26.901

OIT. (2020). ILO Note: Covid 19 and the World of Work: Impacts and Responses. Ginebra: OIT. https://www.ilo.org/wcmsp5/groups/public/---dgreports/--dcomm/documents/briefingnote/wcms_738753.pdf

OIT. (2021). Observatorio de la OIT: La COVID-19 y el mundo del trabajo. Séptima edición. Estimaciones actualizadas y análisis. https://www.ilo.org/global/aboutthe-ilo/newsroom/news/WCMS_767037/lang--es/index.htm

OECD (Organización para la Cooperación y el Desarrollo Económicos). (2020). COVID19 in Latin America and the Caribbean. Tackling coronavirus (COVID-19). Contributing to a global effort. OECD. https://www.oecd.org/coronavirus/policyresponses/covid-19-in-latin-america-and-the-caribbean-an-overview-ofgovernment-responses-to-the-crisis-0a2dee41/

Palomeque, J., Ormaza, J., y Erazo, G. (2021). La Responsabilidad Social en la Dirección de Movilidad del Gobierno Autónomo Descentralizado de Azogues, Ecuador. CIENCIAMATRIA, 7(12), 437-461. https://doi.org/10.35381/cm.v7i12.436

Peñafiel-Chang, L., Camelli, G., \& Peñafiel-Chang, P. (2020). Pandemia COVID-19: Situación política - económica y consecuencias sanitarias en América Latina. CIENCIA UNEMI, 13(33), 120-128. https://doi.org/10.29076/issn.25287737vol13iss33.2020pp120-128p 
Pérez-Rodrigo C., Gianzo, M., Hervás, G., Ruiz, F., Casis, L. y Aranceta, J. (2020) Cambios en los hábitos alimentarios durante el periodo de confinamiento por la pandemia COVID-19 en España. Rev Esp Nutr Com 2020;26(2). DOI 0.14642/RENC.2020.26.2.5213

Pérez, J.M., Espinoza, C., y Peralta, B. (2016). La responsabilidad social empresarial y su enfoque ambiental: una visión sostenible a futuro. Revista Universidad y Sociedad, 8 (3). http://scielo.sld.cu/scielo.php?script=sci_arttext\&pid=S221836202016000300023

Pérez, O.I., Romero, M.C., y Vargas, P. (2020). Interacciones y sinergias entre ODS: un análisis desde la responsabilidad social en Colombia. Revista Desarrollo y sociedad. https://doi.org/10.13043/DYS.86.6

Pérez, B. (2020). Responsabilidad Social Empresarial y Grupos Económicos Familiares en México. Investigación Valdizana, 14(2). pp. 61-71. https://doi.org/10.33554/riv.14.2.707

Petro, J., Diaz, T., Vega, M., Periñan, J., y Maza, J. (2020). Efectos de la implementación de actividades de responsabilidad social empresarial en un supermercado de Cartagena de Indias, Colombia. Revista de jóvenes investigadores Ad Valorem, $3(1)$,

$1-19$. https://revistas.unicartagena.edu.co/index.php/advalorem/article/view/3004

Pilay, F. S., Aguirre, A. A., y Altuna, V. E. (2020). Modelo de gestión de responsabilidad social para distribuidoras de productos agrícolas del cantón Santo Domingo. Visión Empresarial, (10), 31-44. https://doi.org/10.32645/13906852.1006

Pineda, J., Erazo, J., Álvarez, J., y Cárdenas, J. (2021). Impacto de la Responsabilidad Social Empresarial en el Sector Financiero. CIENCIAMATRIA, 7(12), 725-751. https://doi.org/10.35381/cm.v7i12.447

Quevedo, M. R., Patiño, R. J., y Vinueza, S. X. (2021). Responsabilidad social empresarial en el contexto del coronavirus 2019 (COVID-19). Socialium, 5(1), 75-85. https://doi.org/10.26490/uncp.s1.2021.5.1.81

Remache-Rubio, M., Villacis-Torres, S., y Guayta-Toapanta, N. (2018). La responsabilidad social empresarial vista desde un enfoque teórico. Revista Científica Dominio de las Ciencias. ISSN: 2477-8818. http://dx.doi.org/10.23857/dom.cien.pocaip.2017.4.1.enero.550-568 
Ríos, M.A., Guevara, N.D., y Álvarez, J.F. (2020). Pilares fundamentales de la Responsabilidad Social Empresarial en la industria textil en Cuenca - Ecuador. Revista Espacios, 41(1). http://www.revistaespacios.com/a20v41n01/a20v41n01p08.pdf

Rojo-Gutiérrez, M., y Bonilla, D. (2020). COVID-19: La necesidad de un cambio de paradigma económico y social. Cienciamerica, $\quad 9(2), \quad 77-88$. doi:10.33210/ca.v9i2.288

Rosas Arango, S. M., Del Ángel-Caraza, J., y Soriano-Vargas, E. (2020). Infección por COVID-19, una mirada a los factores ambientales relacionados con la pandemia. Revista Nova, 18(35), 101 105. https://doi.org/10.22490/24629448.4193

Saadat, S., Rawtani, D. y Hussain, C. M. (2020). «Environmental perspective of COVID-19». Science of the Total Environment, 728, 138870. DOI: 10.1016/j.scitotenv.2020.13887

Salas, L., García, M. y Azuero, A. R. (2020). Efecto de la responsabilidad social empresarial sobre el posconflicto colombiano: el caso de las pymes. Estudios Gerenciales, 36(154), 80-90. https://doi.org/10.18046/j.estger.2020.154.3547

Sánchez-Gutierrez, F. (2020). Retos pos pandemia en la gestión de residuos sólidos. Cienciamerica, 10 (1). ISSN 1390-9592 ISSN-L 1390-681X. http://dx.doi.org/10.33210/ca.v10i1.354

Severino, P., Villalobos, J., González, N., y Ramírez, R. (2020). Perspectiva de la responsabilidad social empresarial de la salud pública chilena. Revista Venezolana de Gerencia, 25. 1397-1421. http://repositorio.ucm.cl/handle/ucm/3396

Shangzhi, Q., Jianing, J., Jianing, L., Ming-Hsiang, C., \& Xina, Y. (2021, 1 febrero). Can corporate social responsibility protect firm value during the COVID-19 pandemic? ScienceDirect. https://doi.org/10.1016/j.ijhm.2020.102759

Vallejo-Montoya M. E., Carmona-Aricapa M. del R., Vallejo-Ocampo J. D., y RodríguezCastellanos W. A. (2020). La responsabilidad social como estrategia para el desarrollo en contextos de diversidad e inclusión. Latinoamericana de Estudios Educativos, 16(2), 13 - 46. https://doi.org/10.17151/rlee.2020.16.2.3

Vásquez-Giler, M., Zambrano-Vera, T., y Muñoz-Menéndez, M. B. (2020). Responsabilidad social corporativa: estrategia empresarial para el desarrollo 
sostenible. Revista científica multidisciplinaria arbitrada «yachasun», 4(6), 1-7. https://doi.org/10.46296/yc.v4i6.0020

Véliz, D.M., y Zaldumbide, D.A. (2021). Responsabilidad social empresarial en la actividad turística. Una oportunidad para incursionar en los mercados verdes. Polo del Conocimiento, 6 (3). DOI: 10.23857/pc.v6i3.2445

Vergara, A., Olalla, A., Yturralde, J., y Sorhegui, R. (2020). Responsabilidad social corporativa y su impacto en el rendimiento económico de las principales Empresas en Ecuador. Espacios, $41 . \quad$ (13). https://revistaespacios.com/a20v41n10/20411013.html

Vilca-Quispe, W., Loa-Navarro, E., Ramírez-Puraca, A.A., y Medina-Sotelo, C.G. (2020). Responsabilidad social empresarial minera y los conflictos socioambientales en el Perú. Revista Internacional de Investigación en Ciencias Sociales, 17(1). https://doi.org/10.18004/riics.2021.junio.195

Wittmann, L., \& Dantas, D. (2020). Diseño de envases y la pandemia: Cambios en el sector alimentación en Brasil. RChD: creación y pensamiento, 5(9), 1-13. doi:10.5354/0719-837X.2020.57795

Yauri, G.M., Erazo, J.C., Narváez, C.I., y Paulina, V. (2020). Plan de responsabilidad social empresarial para el sector inmobiliario. Revista Arbitrada Interdisciplinaria Koinonía, $5(10)$. http://portal.amelica.org/ameli/jatsRepo/105/1051316014/html/

Zárate-Rueda, R., Beltrán-Villamizar, Y., y González-García, C. (2020). Relacionamiento con stakeholders en el marco de la responsabilidad social empresarial. Revista espacios, 41 http://es.revistaespacios.com/a20v41n32/a20v41n32p20.pdf 